\title{
A rare cause of chronic diarrhoea: a diagnosis to keep in mind
}

\author{
Krzysztof Dąbkowski ${ }^{\circledR 1}$, Honorata Mruk', Karolina Legutko-Pacura', Ligia Gracz-Klata', \\ Jolanta Kunikowska², Teresa Starzyńska ${ }^{1}$
}

${ }^{1}$ Department of Gastroenterology, Pomeranian Medical University, Szczecin, Poland

${ }^{2}$ Nuclear Medicine Department, Medical University of Warsaw, Poland

Key words: gastrinoma; lymph node; diarrhoea; EUS

A 61-year-old male patient, who had undergone surgery for a perforated small bowel ulcer two weeks earlier, reported for an outpatient visit to the gastroenterologist (TS). The patient presented with diarrhoea, weight loss, and weakness.

He had suffered from watery diarrhoea, with 20 to 30 bowel movements daily, for five years. Despite having visited many different specialists, his problems remained unresolved. Common causes of diarrhoea were excluded, including bacterial infection, parasites, colorectal cancer, inflammatory bowel diseases, and celiac disease. Colonoscopy with biopsy $(2 \times)$, computed tomography (CT) of the abdomen $(1 \times)$, and ultrasonography of the abdomen $(3 \times)$ were performed and revealed no abnormality. Meanwhile, the patient lost $30 \mathrm{~kg}$ and was so weak that he could not even walk. His medical history also included severe reflux oesophagitis and duodenal peptic ulcer, with good response to typical treatment 4 years before. His family history was unremarkable.

Clinical examination revealed mild-severe general status, cachexia, paleness, and tachycardia (110 bpm). Standard laboratory tests showed mild anaemia, hypoproteinaemia, and dyselectrolitaemia. Results of other blood tests were within normal ranges.

According to the clinical presentation, gastrinoma was suspected, and high doses of oral esomeprazole (160 mg daily) were administered. Rapid improvement of symptoms with cessation of diarrhoea was achieved. Gastroscopy revealed thickening of gastric folds (Fig. 1). Positron emission tomography (PET/CT) with $\left[{ }^{68} \mathrm{Ga}\right]$ Ga-DOTATATE demonstrated a 10 -mm soft tissue lesion with increased somatostatin receptor expression located close to the duodenum (Fig. 2). This lesion was visualized as an abnormal lymph node $(12.5 \mathrm{~mm})$ in the hepatoduodenal ligament during endoscopic ultrasound (Fig. 3). The patient also had a slightly increased level of gastrin (190 pg/mL; normal level 100 pg/mL).
The patient underwent surgical treatment, and the abnormal lymph node was removed. Histopathological examination revealed typical morphological and immunohistochemical features of a well-differentiated neuroendocrine tumour. The patient has remained symptom free during the 4.5 years since surgery, with a diagnosis of primary lymph node gastrinoma or occult primary gastrinoma with lymph node metastases.

Gastrinoma is a gastrin-producing neuroendocrine tumour that triggers hypersecretion of hydrochloric acid [1]. Most of these tumours arise in the so-called "gastrinoma triangle", delineated by the second and third portions of the duodenum, the head and neck of the pancreas, and the junction of the cystic and common bile ducts [1]. These tumours can also rarely occur in the stomach, lymph nodes, liver, bile duct, ovary, and heart [1]. The existence of true primary lymph node gastrinoma is still an area of debate [2]. What is of special importance is that with

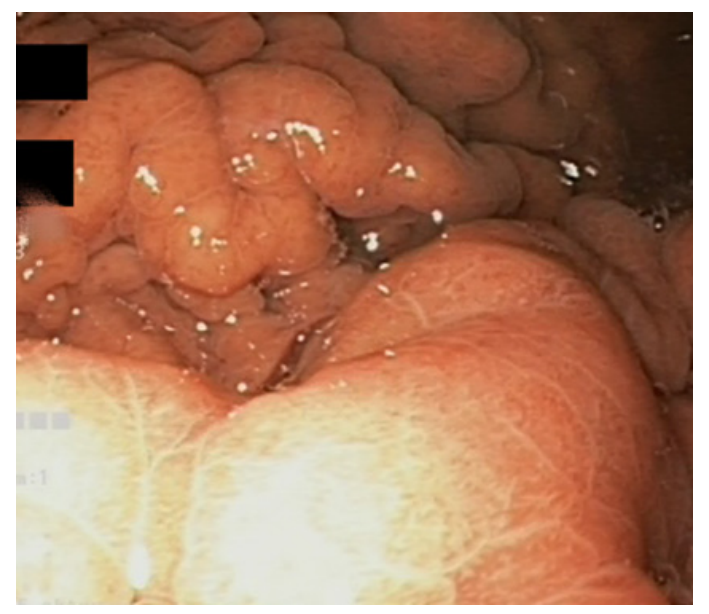

Figure 1. Endoscopy showing thickening of the gastric folds (an endoscopic feature of gastrinoma) 


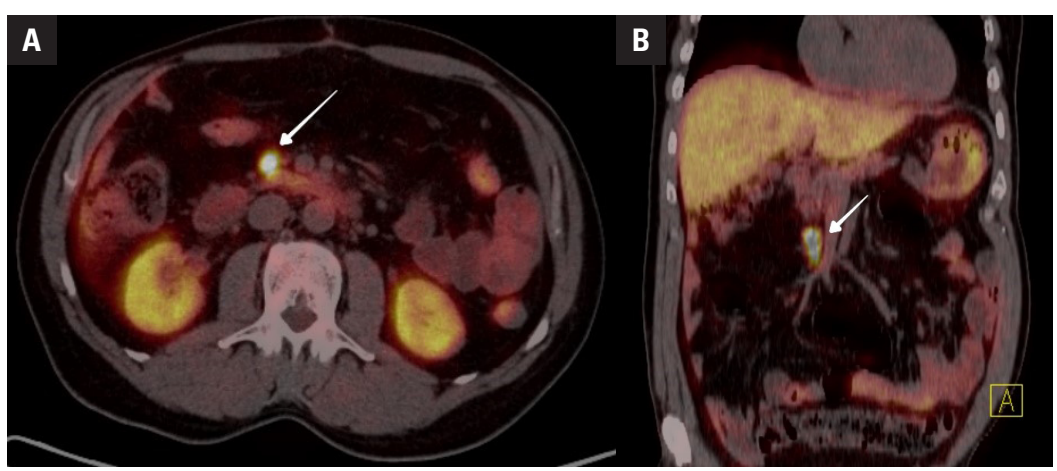

Figure 2. $\left[{ }^{68} \mathrm{Ga}\right] \mathrm{Ga}$-DOTATATE PET/CT showing abnormal uptake near the pancreatic head and duodenal wall

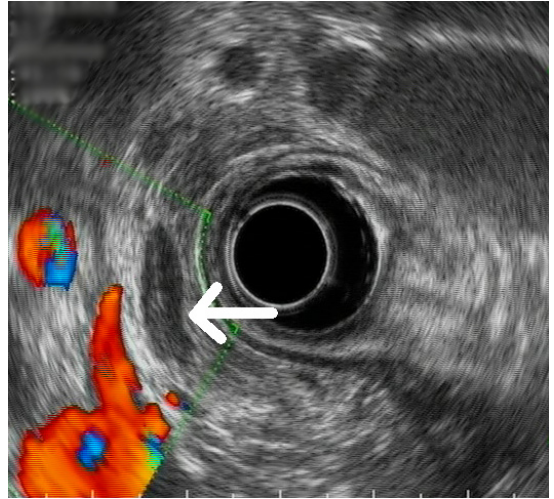

Figure 3. Endoscopic ultrasound showing 12.5-mm lymph node in the hepato-duodenal ligament

suspicion of primary lymph node gastrinoma, the possibility of a metastatic lymph node of a gastrin-producing tumour in an occult location (microgastrinoma) should be considered in the differential diagnosis [3]. The diagnosis is thus rather one of exclusion after long-term observation [2] and an indication for careful exploration of the surgical field, including careful assessment of the gastrinoma triangle and intraoperative sonography, transillumination, and duodenotomy [2,3].

Typical clinical manifestations of gastrinoma include multiple peptic ulcers that are resistant to treatment and in atypical locations, chronic diarrhoea, oesophagitis, and weight loss [1]. The symptoms are often confused with those of other conditions such as indigestion, dyspepsia, or irritable bowel syndrome [4]. Moreover, the use of proton pump inhibitors typically leads to rapid symptom improvement, representing an effective method of symptomatic treatment that carries the risk of masking the clinical course and delaying proper diagnosis for years [1, 4].

From a practical point of view, gastrinoma biochemical diagnostics is based on gastrin serum level, which typically exceeds 10 times the normal concentration, although slightly elevated levels do not exclude the diagnosis [1]. CT, magnetic resonance, endoscopic ultrasound, and PET/CT with ${ }^{68} \mathrm{Ga}$-DOTA-conjugated somatostatin receptor targeting peptides are the modalities used for location diagnostics of the tumour $[1,5]$. In our case, taking into account the typical clinical course, despite only slightly elevated levels of gastrin, the attending physician referred the patient for $\left[{ }^{68} \mathrm{Ga}\right]$ Ga-DOTATATE PET/CT, which has a sensitivity, specificity, and diagnostic accuracy in gastro-entero-pancreatic neuroendocrine tumours reaching 90\% [5]. This imaging showed the exact location of the tumour and paved the way for a correct diagnosis and effective treatment.

Our patient had typical clinical manifestations of gastrinoma, yet despite many gastroenterological appointments, the diagnosis was not established for a long time. During this delay, the patient experienced malabsorption and cachexia. For this reason, we believe that it is important to remind clinicians that one cause of diarrhoea is a gastrin-producing tumour. We emphasize that the gastrinoma location can be atypical and that normal or near-normal levels of serum gastrin do not exclude the diagnosis.

\section{Conflict of interest}

None declared.

\section{References}

1. Lipiński M, Rydzewska G, Foltyn W, et al. Gastroduodenal neuroendocrine neoplasms, including gastrinoma - management guidelines (recommended by the Polish Network of Neuroendocrine Tumours). Endokrynol Pol. 2017; 68(2): 138-153, doi: 10.5603/EP.2017.0016, indexed in Pubmed: 28540972.

2. Teng A, Haas C, Lee DY, et al. Primary lymph node gastrinoma. A diagnosis of exclusion. A case for duodenotomy in the setting of a negative imaging for primary tumor: A case report and review of the literature. Int J Surg Case Rep. 2014; 5(11): 849-852, doi: 10.1016/j.ijscr.2014.08.019, indexed in Pubmed: 25462049.

3. Anlauf M, Enosawa T, Henopp T, et al. Primary lymph node gastrinoma or occult duodenal microgastrinoma with lymph node metastases in a MEN1 patient: the need for a systematic search for the primary tumor. Am J Surg Pathol. 2008; 32(7): 1101-1105, doi: 10.1097/PAS.0b013e3181655811, indexed in Pubmed: 18520436.

4. Basuroy R, Bouvier C, Ramage JK, et al. Delays and routes to diagnosis of neuroendocrine tumours. BMC Cancer. 2018; 18(1): 1122, doi: 10.1186/s12885-018-5057-3, indexed in Pubmed: 30445941.

5. Bozkurt MF, Virgolini I, Balogova S, et al. Guideline for PET/CT imaging of neuroendocrine neoplasms with Ga-DOTA-conjugated somatostatin receptor targeting peptides and F-DOPA. Eur J Nucl Med Mol Imaging. 2017; 44(9): 1588-1601, doi: 10.1007/s00259-017-3728-y, indexed in Pubmed: 28547177. 